\title{
REGION BASED CLUSTERING FOR DATA COLLECTION IN WSN
}

\author{
Ramandeep Kaur (1), Dinesh Kumar (2) \\ (1) Research Scholar, Department of Computer Science \& Engineering, GZSCCET, Bathinda \\ (2) Associate Professor, Department of Computer Science \& Engineering, GZSCCET, Bathinda
}

\section{ABSTRACT}

The lower cost and easier installation of the WSNs than the wired counterpart pushes industry and academia to pay more attention to this promising technology. Large scale networks of small energy-constrained sensor nodes require techniques and protocols which are scalable, robust, and energy-efficient. The most efficient approach provided by clustering the nodes is hierarchy. The one node will send the data to another node and the another node will send to its neightbouring node. In smart cities, wireless sensor networks (WSNs) act as a type of core infrastructure that collects data from the city to implement smart services. Our thesis work included the region based clustering, cluster head selection and energy efficient communication using static base station and movable mobile nodes. Since it was earlier proposed that clustering improves the network lifetime. We modified the region based clustering by dividing the network area into $\mathrm{n}$ regions with cluster head chosen for each region and proposed a new method for cluster head selection having less computational complexity. It was also found that the modified approach has improved performance to that of the other clustering approaches. We have used the mobile nodes for each section with controlled trajectory path as a reference to compare the performance of each of the clustering methods.

\section{Keywords}

Wireless0 Sensor Networks, Protocol, Cluster Head, Base Station, Mobile node.

\section{INTRODUCTION}

Wireless technology has expanded the boundaries of our world by multiple leaps. Through this innovation, humans are given freedom to work off from their desks or perhaps outside. The new freedom that human are getting down to work with their computers has started creating the globe of technology. Though a young technology, the applications are varied and promise to be even additional varied. These networks area unit collections of little devices, called motes, with restricted processing and power. Every sensor node has processing power close to 1-100th of the computing power of a PDA, however once combined with many alternative motes, they mix to create a robust network system. Wireless sensor Networks, or WSNs, are used for analysing the changes in data and change higher knowledge assortment in scientific studie and produce simpler results. Application area like strategic military defenses, pinpoint and the origin of a shooting and its monitoring are covered inside WSN. All of those uses rely on the power to gather knowledge like lightweight, vibration, moisture, temperature, and more, likewise because the ability to speak with one another. This last ability is what makes a set of nodes most additional powerful network. Recent advances in sensing, computing and communication technologies as well as the necessity to unendingly monitor physical environement have led to the study and development of Wireless sensor Networks (WSNs). WSN comprises four main components: A radio, a processor, sensors and battery. A WSN is created by multiple tiny sized deployed sensor device nodes in different application spaces. In most deployments, the device sensor nodes have self-organizing capabilities, to create associate structure known as cluster so as to collaboratively perform a specific task. Wireless sensor Networks are found appropriate for applications like police investigation, agriculture, smart homes, automation, vehicle traffic management, environment observation, and disaster detection. WSN is nice technology which will revolutionize in each and every sector and sphere of our lives. In fact, it's the potential to considerably amendment the method we tend to live - similar to the net and World Wide internet - maybe a lot of thus. Of course, WSN can exploit the net and WWW; it'll connect the physical world to the net at fine graininess. the facility of WSN lies in making a pervasive setting capable of remote sensing, observation and management. The positive advantages of this are quite obvious; such a technology are going to be a can accomplish fine graininess chase of what's occurring at distant and usually in inaccessible locations.

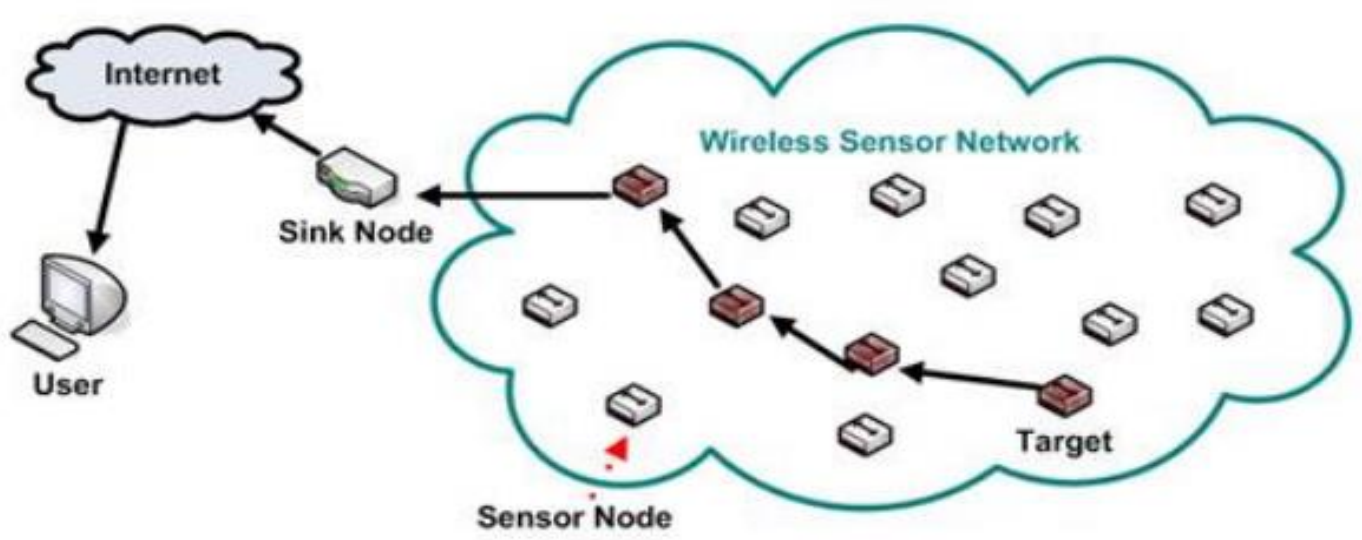

Figure 1. Wireless Sensor Network 


\section{CHARACTERISTICS OF WIRELESS SENSOR NETWORK}

- Infrastructure less: In wireless sensor network each and every sensor node can communicate with each other without any fixed infrastructure. There is very less overhead in communication.

- Mobility: Mobility of nodes in wireless sensor networks is less. Due to this kind of feature they are used in event tracking purposes like motion detection of video frames etc.

- Multi-Hoping: Wireless sensor Networks composed of several nodes and they are communicating with each other and describe several paths to several node. Here actually the packet traverses from one node to another node to reach the destination through several paths. Due to this Multi-hop features energy associated with each node can be conserved [4].

- Openness: Wireless sensor network access information and services regardless of Geographic position.

- Network size: In wireless sensor network the node are scattered in large geographical area.

- Homogeneous Network: A Wireless sensor network composed of homogeneous devices that is node has same features [2].

- Addressing scheme: In wireless sensor networks, the addresses contain the pair of attributes associated with their values. This pair of value specifies certain physical parameters to be sensed, stored and to be communicated to other sensor nodes. [1].

- Location Awareness: Every sensor node knows its own position means it knows the $x$ and y coordinates in a 2 dimensional space. Data is collected according to the location of the sensor node.

- Reliable transmission of Data: To have a proper request-response model the node should request to a proper node .This node should process the request efficiently and reply as soon as possible. So that the mechanism should be reliable.

- Fault tolerant: In wireless sensors, if one node fails then it does not affect the network operation because there are other adjacent nodes collecting similar data. The accuracy of data collected is reduced.

\section{CHALLENGES IN WIRELESS SENSOR NETWORK}

The various challenges in wireless sensor network have got raised when they are implemented in various fields and industrial sectors. There are so many controllable and uncontrollable parameter [4] by which the implementation of wireless sensor network affected such as.

- Energy conservation: In wireless sensor network every node is equipped with sensor and the sensor devices are in working condition depending upon the power supplied by attached battery. To have better performance the network should operate for large time. As we know that the wireless sensor node has small size and because of this small size we cant fit the bigger battery inside the sensor node and thereby it has very less energy available for its functioning. It will be difficult for refilling and replacing the batteries and will be very costlier process. Therefore we need some energy efficient and robust protocol that increases the overall lifetime of the wsn network and also helps in increasing the overall throughput and efficiency of the network.

- Operation in antagonistic environment: Sensor network can be operating in antagonistic environmental condition. So design issues of sensor node are carefully considered. The protocol used for the sensor network should be robust which can work inside harsh conditions. If any fault occurs, it should have the capability to tolerate and recover the faults automatically.

- Communication quality: Sensor network have very low quality communication depending upon different situation like when it operated in some unpleasant environment then communication quality is very poor. It is environment specific.

- Availability of resources: When the resources required by sensor network are unavailable then the sensor networks try hard to provide the desired QoS.

- Data processing: Data collected by many sensors may contain redundant data. So data aggregation required in network processing so that redundant data can't be transmitted number of time. It will help to conserve some amount of energy for further transmission.

- Scalability: Wireless sensor node are composed of large number of sensor node and many more nodes can be added in design stage [2].

\section{APPLICATIONS IN WIRELESS SENSOR NETWORK:}

- Intrusion Detection: Due to presence of less mobility and stationary property of wireless sensor network can be used to track object event. It can be used for security purposes that are for surveillance purposes. Generally high resolution camera is equipped with sensors that can be used to form a network that monitor a restricted area access. If any outsider enter into this region without any proper authentication then some sort of signaling event occur it may be an alarm message quickly propagate to a handling authority [2].

- Avalanche prediction: Movement of large snow masses can be predicted by device equipped with sensor. GPS one of the detecting devices use for this purposes [2]. 
- Environment Observation and Forecasting System: The Environment Observation and Forecasting System (EOFS) is a distributed system that spans large geographic areas and monitors, models and forecasts physical processes such as environmental pollution, flooding, among others. It consists of three components: sensor stations, a distribution network, and a centralized processing farm [1].

\section{RESEARCH GAP}

Due to the deployment of WSNs in unattended harsh environment conditions, it gets to be unrealistic to charge or replace their batteries. Accordingly, the energy efficient operation of wireless sensors is very important to prolong the lifetime of overall wireless sensor networks. Furthermore, in WSN, there is wireless communication among all the nodes. Moreover, the energy consumed to transmit a message is exceptionally more than the energy needed to receive the same message. Besides all this, the route of each message destined to the base station too decides the network lifetime; for example, any node with depleted battery in short routes to the base station may lead to decreased network lifetime. While, using a long route composed of many sensor nodes can significantly increase the network delay.

- To decide cluster area on the basis of chosen cluster head consumes huge amount of energy resources in the communication between nodes for the purpose of fixing the cluster region and its head. When two cluster heads are very close to each other that means their ranges will overlap each other which will create a major problem.

- $\quad$ No parameter has been explained for the election of Cluster head. There are several parameters that are involved in the election of cluster head.

- Same level of power is used to amplify the signals from member node to cluster head, cluster head to mobile node and mobile node to base station. This will consume lot of energy.

- Mobile node requires large amount of energy for transferring the data of complete sector to the base station.

\section{OBJECTIVES}

- To review the existing routing algorithms in wireless sensor networks.

- To propose an enhanced energy efficient algorithm with maximum life span and throughput.

- To introduce the various levels of power for signal amplification.

- To elect the cluster head based on various parameters like maximum residual energy, minimum separation distance and minimum distance to mobile node.

- $\quad$ To simulate the proposed algorithm in Matlab environment and evaluate the results with the existing work.

\section{METHODLOGY}

- Sensor nodes are randomly deployed in the network. Different network areas have been used for experimental purposes. The base station is located outside the network.

- $\quad$ All nodes sends its energy level and location information to the sink, which divides network into clusters and further divided into sectors based on number of cluster groups. Each sector is assigned a mobile node.

- $\quad$ The $\mathrm{CH}$ is selected by using hybrid model of both centralized and distributed election mechanism. The cluster head election mechanism will include various parameters like maximum residual energy of a node, minimum separation distance and minimum distance to the mobile node. Each $\mathrm{CH}$ will create a TDMA schedule for the member nodes to transmit the data.

- Nodes will have various level of power for signal amplification. The three levels of power are used for amplifying the signal. As the member node will send only its own data to the cluster head, the power level of the member node is set to low. The cluster head will send the data of the whole cluster to the mobile node, therefore the power level of the cluster head is set to medium. High power level is used for mobile node which will send the data of the complete sector to the base station. Using low energy level for intra cluster transmissions (within the cluster) with respect to cluster head to mobile node transmission leads in saving much amount of energy. Moreover, multi-power levels also reduce the packet drop ratio, collisions and/ or interference for other signals.

\section{RESULTS AND DISCUSSSIONS}

This section presents the simulation results of the work done and the proposed approach. The proposed approach has been implemented in MATLAB. The parameters required for the simulation is shown in the following Table 1.

Table 4.1 Simulation Parameters

\begin{tabular}{|c|c|}
\hline Network Parameters & Values \\
\hline Network Size & $80 \mathrm{~m}^{\star} 80 \mathrm{~m}$ \\
\hline Number of Sensor Nodes & 100 \\
\hline
\end{tabular}




\begin{tabular}{|c|c|}
\hline Initial Energy of Sensor Nodes & $0.5 \mathrm{~J}$ \\
\hline Packet Size & $4000 \mathrm{bits}$ \\
\hline Data Aggregation Energy consumption & $5 \mathrm{~nJ} / \mathrm{bit}$ \\
\hline Amplification Energy (Cluster to BS) $\mathrm{d}>=\mathrm{d} 0$ & $\mathrm{Efs}=10 \mathrm{pJ} / \mathrm{bit} / \mathrm{m} 2$ \\
\hline Amplification Energy (Cluster to BS) $\mathrm{d}<=\mathrm{do}$ & Emp $=0.0013 \mathrm{pJ} / \mathrm{bit} / \mathrm{m} 2$ \\
\hline
\end{tabular}

\section{NEWTORK MODEL}

The network area is of $80 * 80 \mathrm{~m}$. The network area is divided into small regions of $40 * 40 \mathrm{~m}$ each. Each region has its own cluster head. The base station is fixed. There are 4 mobile nodes for each sector. Each sector will have 4 clusters inside it. Each mobile node will move along the boundary of the sector network. Figure 2 explains the network model of size $80^{\star} 80$ $\mathrm{m}$ with 16 regions and 4 sectors. Each sector is having its own mobile node marked with pink color. The base station is available on the top of the network.

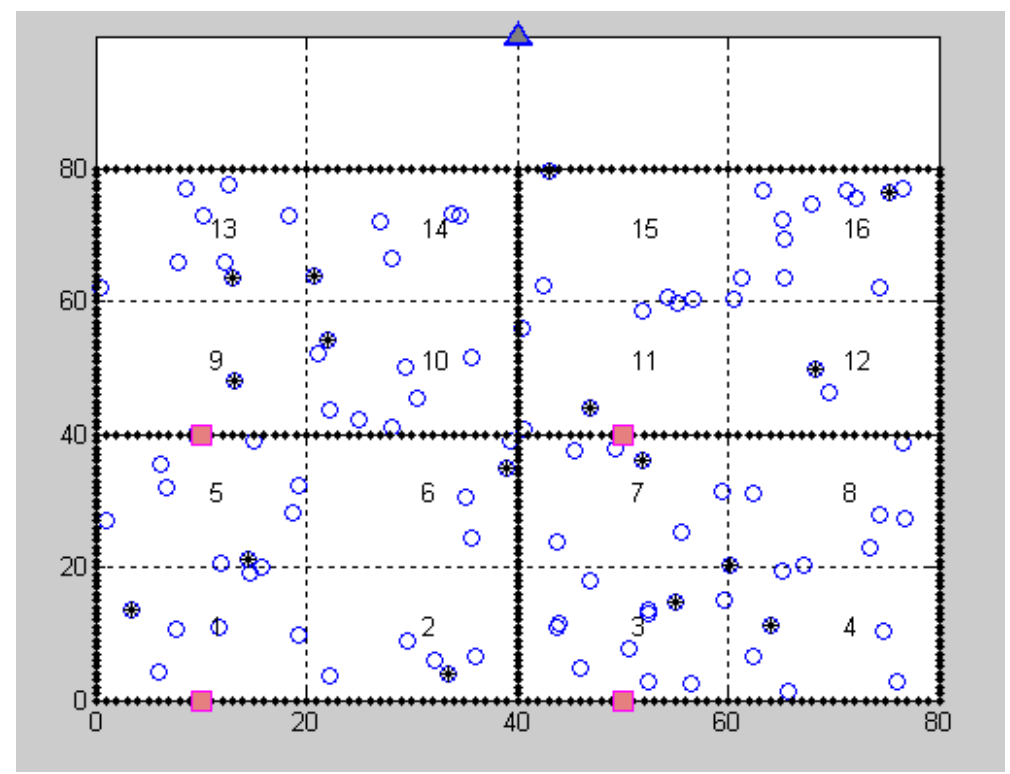

Figure 2. Network Model

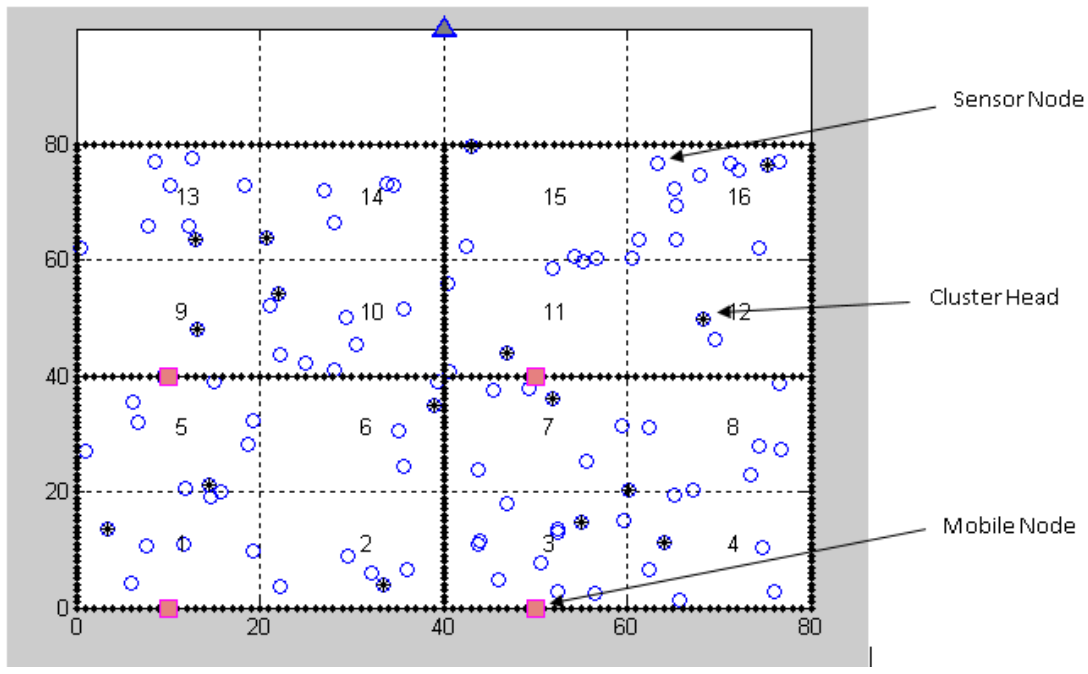

Figure 3. Network when nodes are Allive

\section{PERFORMANCE METRICS}

The performance metrics used in proposed work compared with the existing work are network life time, throughput, average energy consumption and average remaining energy. 


\section{NETWORK LIFE TIME}

The lifetime of network depends upon the number of Dead \& Alive Nodes. The proposed scheme plays an important role to achieve better network life time. Figure 4 \& Figure 5 clearly represents the longer stability of the proposed scheme. An equal energy is consumed by each node in each round and some nodes get dead with variation of time. The proposed scheme achieves more stability period and longer network lifetime.

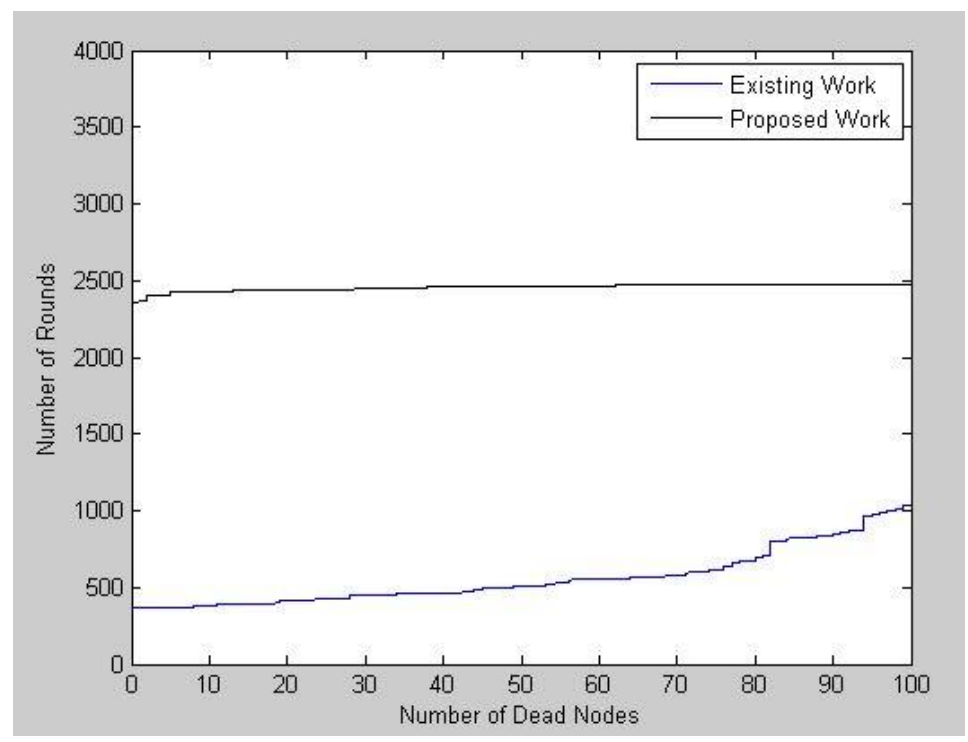

Figure 4. Analysis of dead nodes

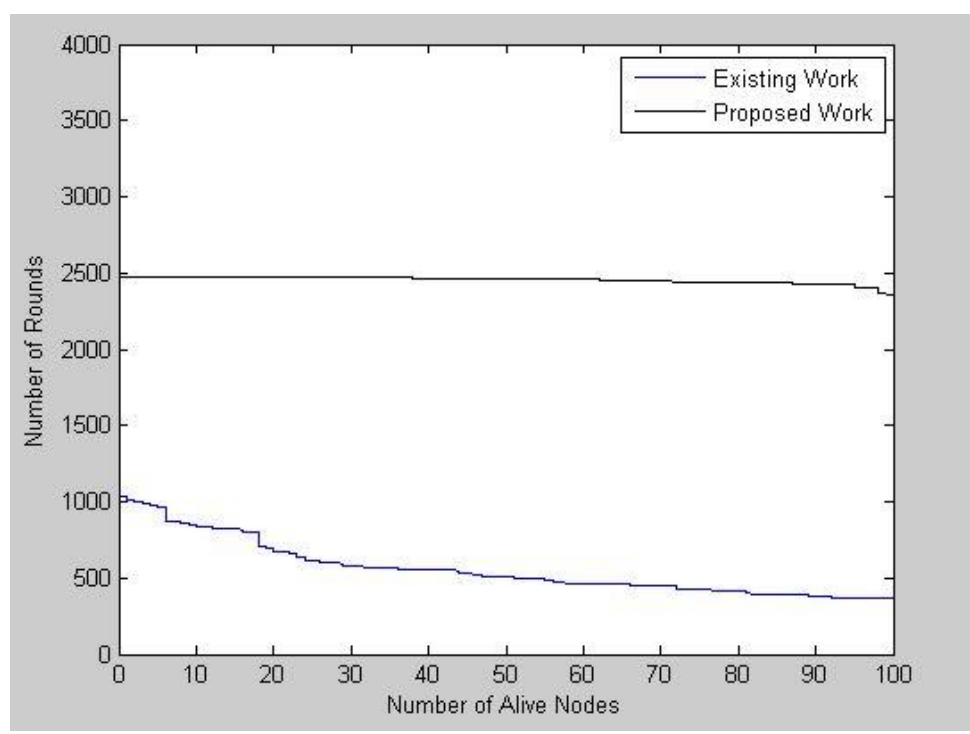

Figure 5. Analysis of Allive nodes

\section{THROUGHPUT}

The throughput is the fundamental parameter which checks the efficiency of the network in terms of data transfer. It is calculated as the successful number of message delivered over a communication channel and is measured in bits per second(bps).It represents the number of packets received by each node in the network over the time interval taken due packets delivery. Throughput is measured as:

$$
\text { Throughput }=\sum \frac{\text { Number of all delivered packets }}{\text { Receiving time interval length }}
$$

As illustrated in Figure 6 the throughput comparison of existing and proposed algorithm. Throughput is also defined as the packets that successfully reached at the sink which means that the data received at sink is maximum with less packet dropping. The proposed algorithm achieves high throughput than existing algorithm as shown. Number of packets received by sink depends upon the number of Alive nodes present in the network. More the Alive nodes, large number of packets are received by the destination sink node which improves the overall throughput of the network. 


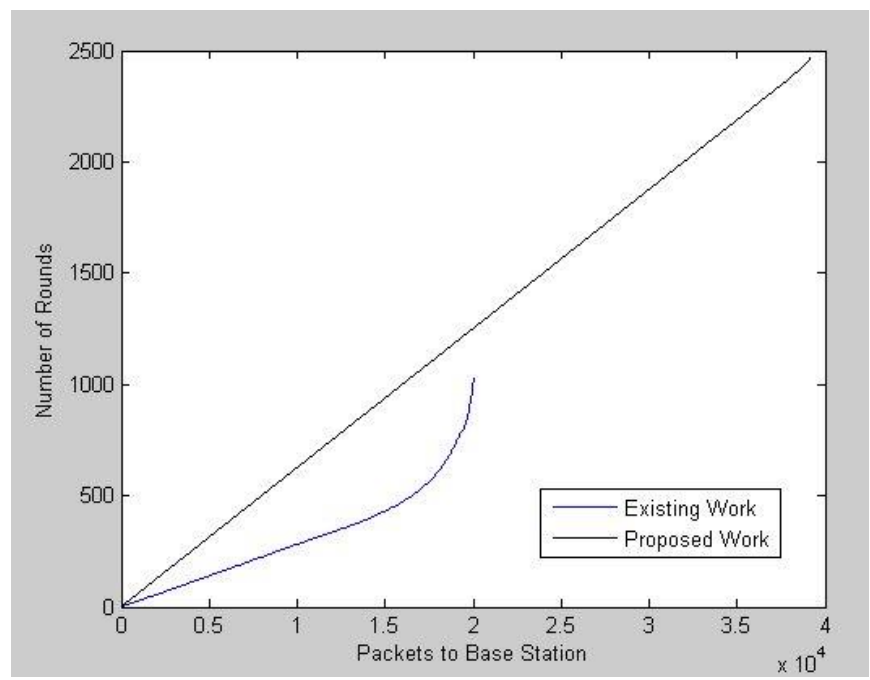

Figure 6. Packets sent to the base station

\section{ENERGY CONSERVATION}

Figure 7 represents the average energy consumed by the network during each round of the transmission time. The average energy consumed by the proposed algorithm is minimum as compared to the existing algorithm.

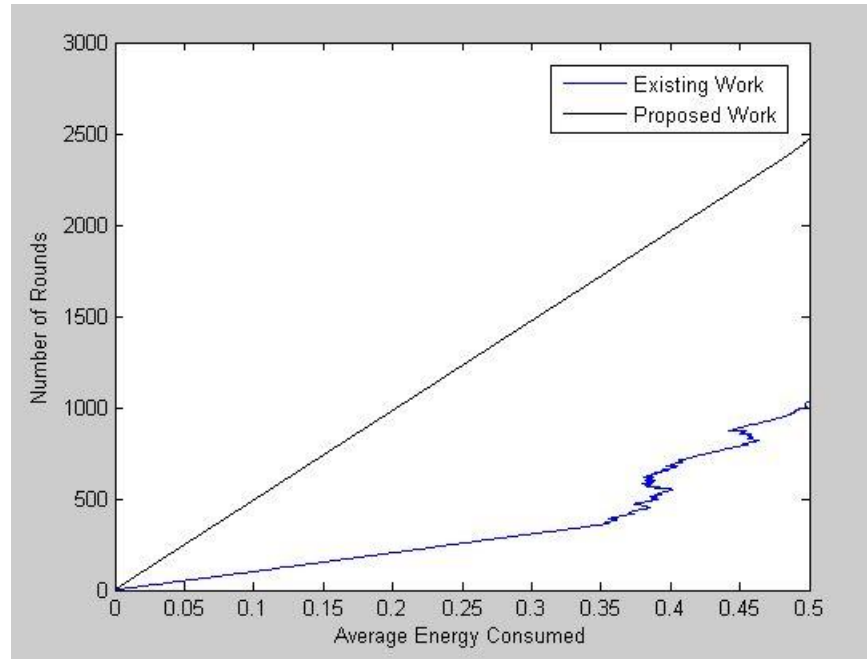

Figure 7. Energy consumed by the nodes

We can see that almost all the sensors are alive for the proposed algorithm for quite long period of time, hence extending the network efficiency. For existing algorithms, as no. of dead sensors will increase, their efficiency will decrease.

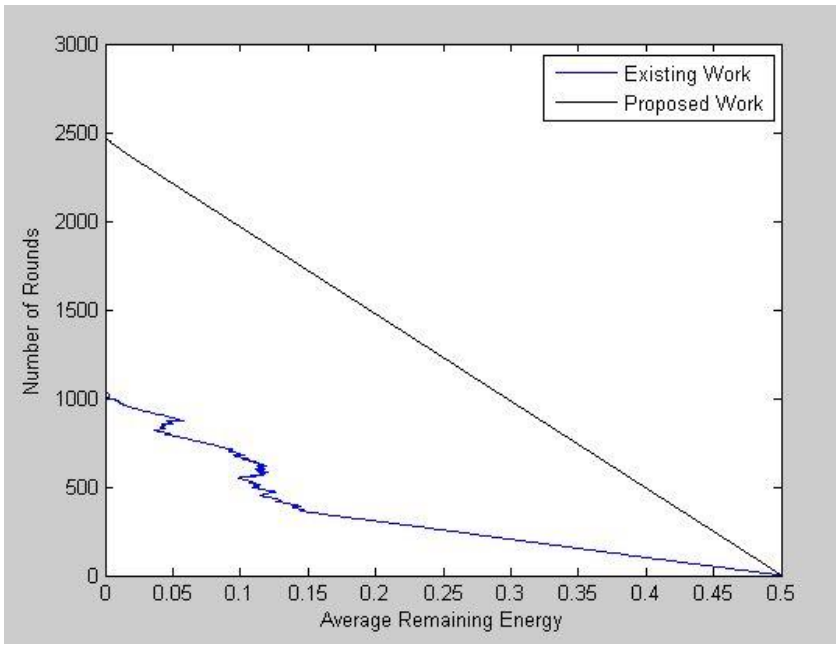

Figure 8. Average Remaining Energy 


\section{CONCLUSION}

We modified the region based clustering by dividing the network area into $\mathrm{n}$ regions with cluster head chosen for each region and proposed a new method for cluster head selection having less computational complexity. It was also found that the modified approach has improved performance to that of the other clustering approaches. We have used the mobile nodes for each section with controlled trajectory path as a reference to compare the performance of each of the clustering methods. It was found that the proposed algorithm gives a much-improved network lifetime as compared to existing work. In this thesis, the nodes are divided into multiple regions depending upon the network area. The region height and width can be controlled according to the requirement of the network. We virtually divide the network into regions according to the proximity to the BS denoting the closest region as the boundary zone and the farthest region as the non-boundary zone. The nodes have been classified according to the region that they fall in. Based on our model, transmission of packets for cluster-based WSNs has been proposed to balance the load among cluster heads that fall in different regions. Multiple transmission power levels have been used for energy saving architecture. This algorithm is applied prior to a cluster algorithm to improve the performance of the clustering algorithm without affection the performance of individual sensor nodes. As a result, the network lifetime has been prolonged. By analyzing the results and graphs, we have reached up to the solution that by changing the clustering and communication mechanism, the overall lifetime of the complete network can be improved. In all of the methods discussed above the energy parameter is taken into consideration only during cluster head selection. The performance may be increased by considering energy as a parameter during clustering itself. Multiple number of experiments have been conducted to evaluate the effectiveness of the experiments.

\section{REFERENCES}

[1] Y. Xiuwu, Fan Feisheng Zhou Lixing and Z. Feng, "WSN Monitoring Area Partition Clustering Routing Algorithm for Energy-Balanced," IEEE, pp. 80-84, 2016.

[2] S. Bera, S. Misra, Sanku Kumar Roy and Mohammad S. Obaidat, "Soft-WSN: Software-Defined WSN Management System for loT Applications," IEEE, pp. 1-8, 2016.

[3] N. A. M. Alduais, J. Abdullah, J. Abdullah, A. Jamil and L. Audah, "An Efficient Data Collection and Dissemination for IOT based WSN," IEEE, 2016.

[4] O. Singh, V. Rishiwal and M. Yadav, "Energy Trends of Routing Protocols for H-WSN," IEEE, 2016.

[5] R. Kumari and. P. Nand, "Performance Comparison of various Routing Protocols in WSN and WBAN," IEEE, pp. 427-431, 2016.

[6] Hector Kaschel and ohanna Ortega , "Energy efficiency in routing protocols applied to WSN," IEEE, 2016.

[7] Asdianur Hadi and Ida Wahidah, "Delay Estimation using Compressive Sensing on WSN IEEE 802.15.4," IEEE, pp. 192-197, 2016.

[8] Mohd Zaki Shahabuddin, Halabi Hasbullah and Izzatdin A Aziz, "eliminary Framework of Topology Control Algorithm Ahieve Node's Energy Efficiency," IEEE, pp. 259-263, 2016.

[9] Abhaykumar L. Gupta and Narendra Shekokar, "A Novel Approach to Improve Network Lifetime in WSN by Energy Efficient Packet Optimization," IEEE, 2016.

[10] B. Bengherbia, S. Chadli, M. Ould Zmirli and A. Toubal, "A MicroBlaze Based WSN Sink Node Using XBee Transceiver," IEEE, pp. 831-834, 2016.

[11] Gagandeep Kaur, Deepali and Rekha Kalra, "Improvement and Analys Security of WSN From Passive Attack," IEEE, pp. 4520-425, 2016.

[12] M. Wu, H. Liu and Q. Min, "Lifetime Enhancement by Cluster Head Evolutionary Energy Efficient Routing Model for WSN," IEEE, pp. 545-548, 2016.

[13] Roman Lara-Cueva, Rodolfo Gordillo, Liliana Valencia and Diego S. Ben, "Determining the Main CSMA Parameters for Adequate Performance of WSN for Real-time Volcano Monitoring System Applications," IEEE, pp. 1-9, 2016.

[14] Sanaa. S. Abd El dayem and M. R. M. Rizk , "An Efficient Authentication Protocol and Key Establishment in Dynamic WSN," IEEE, pp. 178-182, 2016.

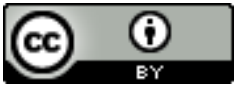

This work is licensed under a Creative Commons Attribution 4.0 International License.

DOI:10.24297/ijct.v16i5.6251 